\title{
On Fractional Orthonormal Polynomials of a Discrete Variable
}

\author{
I. Area, ${ }^{1}$ J. D. Djida, ${ }^{2}$ J. Losada, $^{3}$ and Juan J. Nieto ${ }^{3,4}$ \\ ${ }^{1}$ Departamento de Matemática Aplicada II, E.E. Telecomunicación, Universidade de Vigo, 36310 Vigo, Spain \\ ${ }^{2}$ African Institute for Mathematical Sciences (AIMS), Limbe Crystal Gardens, P.O. Box 608, Southwest Region, Cameroon \\ ${ }^{3}$ Facultade de Matemáticas, Universidade de Santiago de Compostela, 15782 Santiago de Compostela, Spain \\ ${ }^{4}$ Faculty of Science, King Abdulaziz University, P.O. Box 80203, Jeddah 21589, Saudi Arabia
}

Correspondence should be addressed to Juan J. Nieto; juanjose.nieto.roig@usc.es

Received 25 March 2015; Accepted 10 June 2015

Academic Editor: Guang Zhang

Copyright (C) 2015 I. Area et al. This is an open access article distributed under the Creative Commons Attribution License, which permits unrestricted use, distribution, and reproduction in any medium, provided the original work is properly cited.

A fractional analogue of classical Gram or discrete Chebyshev polynomials is introduced. Basic properties as well as their relation with the fractional analogue of Legendre polynomials are presented.

\section{Introduction}

Fractional calculus, in which derivatives, differences, integrals, and sums of fractional order are defined and studied, is nearly as old as the classical calculus of integer order [1]. Its origin goes back to L'Hôpital and Leibniz in 1695 [2] and mathematicians like Fourier, Euler, Laplace, Riemann, and Liouville have made key contributions to it. Fractional calculus has recently been found wide applications in many areas of science and engineering as viscoelastic systems, fluid dynamics, or solid dynamics, to cite some of them [3].

Moreover, orthogonal polynomials and special functions appear in many problems of pure and applied mathematics as, for example, numerical quadrature, least-squares method of approximation, and queueing theory or optics, just to cite a few of them. The systematic analysis of their properties goes back to the XVIII century, in the framework of some problems appearing in celestial mechanics [4]. We refer to [59] as basic references on this topic.

Very recently, the problem of numerical evaluation of a fractional integral with unit upper integration limit,

$$
\frac{1}{\Gamma(\alpha)} \int_{0}^{1} f(\tau)(1-\tau)^{\alpha-1} d \tau
$$

has been considered in [10] by using quasi-polynomial of order $n$ with commensurate power $\beta$,

$$
P_{n}\left(t^{\beta}\right)=P_{n, \beta}(t)=\sum_{k=0}^{n} a_{n, k} t^{k \beta},
$$

giving rise to an orthogonality concept and a new family of orthogonal quasi-polynomials. Moreover, quasi-polynomials orthogonal with respect to fractional densities have been receently introduced in [11].

The main objective of this paper is to introduce a discrete analogue of the quasi-orthogonal polynomials introduced in [10]. In doing so, the paper is structured as follows. In Section 2, we recall the basic definitions and notations. In Section 3, we introduce the fractional Gram orthonormal polynomials. Finally, in Section 4, some numerical tests are presented.

\section{Basic Definitions and Notations}

Next, we recall some basic facts from the theories of orthogonal polynomials and fractional calculus.

2.1. Classical Legendre and Gram Polynomials. One of the simplest and oldest families of orthogonal polynomials is 
the Legendre polynomials $P_{n}(x)$ which can be defined in terms of a Gauss hypergeometric series as

$$
\begin{aligned}
P_{n}(x) & ={ }_{2} F_{1}\left(\begin{array}{c}
-n, n+1 \\
1
\end{array} \mid \frac{1-x}{2}\right) \\
& =\sum_{k=0}^{n} \frac{(-n)_{k}(n+1)_{k}}{(k !)^{2}}\left(\frac{1-x}{2}\right)^{k},
\end{aligned}
$$

where $(A)_{s}$ denotes the Pochhammer symbol, $(A)_{0}=1$, and $(A)_{s}=A(A+1) \cdots(A+s-1)$, and satisfy the orthogonality relation:

$$
\int_{-1}^{1} P_{n}(x) P_{m}(x) d x=\frac{1}{2 n+1} \delta_{n, m}
$$

where $\delta_{n, m}$ denotes the Kronecker delta. We will also consider the orthonormal shifted Legendre polynomials, for which the orthogonality relation is over $[0,1]$; that is,

$$
\begin{aligned}
R_{n}(x) & =\sqrt{2 n+1} P_{n}(1-2 x) \\
& =\sqrt{2 n+1}{ }_{2} F_{1}\left(\begin{array}{cc}
-n, n+1 & \\
1 & x
\end{array}\right) .
\end{aligned}
$$

Then, the first few shifted Legendre orthonormal polynomials are

$$
\begin{aligned}
& R_{0}(x)=1, \\
& R_{1}(x)=\sqrt{3}(1-2 x), \\
& R_{2}(x)=\sqrt{5}(6(x-1) x+1) .
\end{aligned}
$$

Let $\left\{G_{n}(x ; N)\right\}$ be a sequence of polynomials orthonormal with respect to a constant weight function at $N$ equidistant points:

$$
\sum_{x=0}^{N} G_{n}(x ; N) G_{m}(x ; N)=\delta_{n, m} .
$$

Such a sequence exists $[12,13]$ and they seem to be the first orthogonal polynomials of a discrete variable introduced in the literature. Properties about their zeros have been obtained in [14]. Nowadays, they are called either the discrete Chebyshev polynomials, in the terminology of $[15,16]$, or the Gram polynomials [17], in the terminology of [18, 19]. Gram polynomials can be expressed in terms of hypergeometric series as

$$
\begin{aligned}
G_{n} & (x ; N) \\
& =\sqrt{\frac{(2 n+1)(N-n+1)_{n}}{(N+1)_{n+1}}}{ }_{3} F_{2}\left(\begin{array}{c}
-n, n+1,-x \\
1,-N
\end{array}\right), \\
& =\sqrt{\frac{(2 n+1)(N-n+1)_{n}}{(N+1)_{n+1}}} \sum_{k=0}^{n} \frac{(-n)_{k}(n+1)_{k}(-x)_{k}}{(k !)^{2}(-N)_{k}}, \\
n=0,1, \ldots, N . &
\end{aligned}
$$

The following limit relation between Gram polynomials and shifted Legendre orthonormal polynomials holds [7]:

$$
\lim _{N \rightarrow \infty} \sqrt{N} G_{n}(N x ; N)=R_{n}(x) .
$$

2.2. Fractional Integrals, Derivatives, Sums, and Differences. Next, we recall the definitions of fractional integral, derivative, sum, and difference. We would like to notice here that as compared with the long and rich history of fractional calculus [20-23], discrete fractional calculus attracted the interest of researchers only in a short period of time [24-30].

Definition 1 . Let $f: \mathbf{R} \rightarrow \mathbf{R}$ be a given function. The fractional integral of order $\alpha>0$ of $f$ is defined as [20,31]

$$
I^{\alpha} f(t)=\frac{1}{\Gamma(\alpha)} \int_{0}^{t}(t-s)^{\alpha-1} f(s) d s, \quad t>0 .
$$

We would like to notice that for $\alpha<1$ the integral may be singular, but it is well defined if we assume, for example, $f \in L_{\text {loc }}^{1}(\mathbf{R})$.

Definition 2. Let $\alpha \in(0,1)$. The fractional Riemann-Liouville derivative of order $\alpha$ of $f$ is defined as [20,31]

$$
\begin{aligned}
{ }^{\mathrm{RL}} D^{\alpha} f(t) & =D^{1}\left(I^{1-\alpha} f\right)(t) \\
& =\frac{1}{\Gamma(1-\alpha)} \frac{d}{d t} \int_{0}^{t}(t-s)^{-\alpha} f(s) d s,
\end{aligned}
$$

provided the right hand side is defined for almost every $t \epsilon$ $\mathbf{R}^{+}$. The fractional Riemann-Liouville derivative of order $\alpha$ is well defined if, for example, $f$ is absolutely continuous on every compact interval of $\mathbf{R}$.

There are several definitions of fractional integral and fractional derivative [23]. We are not giving a complete list but recall the Caputo fractional derivative [20, 31].

Definition 3. The Caputo fractional derivative is defined as

$$
{ }^{C} D^{\alpha} f(t)={ }^{R L} D^{\alpha} g(t),
$$

with $g(t)=f(t)-f(0)$.

In addition, if $f$ is an absolutely continuous function on every compact interval (of $\mathbf{R}$ ), we can write for $\alpha \in(0,1)$

$$
\begin{aligned}
{ }^{C} D^{\alpha} f(t) & =I^{1-\alpha} D^{1} f(t) \\
& =\frac{1}{\Gamma(1-\alpha)} \int_{0}^{t}(t-s)^{-\alpha} f^{\prime}(s) d s .
\end{aligned}
$$

Definition 4 (see [28]). Let $\alpha>0$; the fractional sum of $f$ of order $\alpha$ with base point $a \in \mathbf{Z}$ is defined by

$$
\Delta_{a}^{-\alpha} f(t)=\frac{1}{\Gamma(\alpha)} \sum_{s=a}^{t-\alpha}(t-s-1)^{(\alpha-1)} f(s),
$$

where $f$ is a function defined for $s=a \bmod 1$ and the falling factorial power function is defined, for $\alpha \in \mathbf{R}$, as [32]

$$
t^{(\alpha)}=\frac{\Gamma(t+1)}{\Gamma(t+1-\alpha)} .
$$

Thus, $\Delta_{a}^{-\alpha} f$ is a function defined for $t=(a+\alpha) \bmod 1$. 
In particular, $\Delta_{a}^{-\alpha}$ maps functions defined on $\mathbf{N}_{a}$ to functions defined on $\mathbf{N}_{a+\alpha}$, where $\mathbf{N}_{t}=\{t, t+1, t+2, \ldots\}$.

Let $n \in \mathbf{N}$ and $\Delta^{n}$ be the well known forward difference operator of order $n$; that is,

$$
\Delta^{n} f(s)=\sum_{k=0}^{n}(-1)^{n-k}\left(\begin{array}{l}
n \\
k
\end{array}\right) f(s+k) .
$$

Definition 5 (see [33], Definition 2.3). Let $\alpha>0$ and $n-1<$ $\alpha \leq n$, where $n=\lceil\alpha\rceil$. The fractional Caputo type difference of order $\alpha$ of $f$ is defined as

$$
\begin{aligned}
{ }^{C} \Delta_{a}^{\alpha} f(t) & =\Delta_{a}^{-(n-\alpha)} \Delta^{n} f(t) \\
& =\frac{1}{\Gamma(n-\alpha)} \sum_{s=a}^{t-n+\alpha}(t-s-1)^{(n-\alpha-1)} \Delta^{n} f(s),
\end{aligned}
$$

for all $t \in \mathbf{N}_{a+\alpha}$.

We will use $\Delta$, to denote both differences and sums. More precisely, $\Delta_{a}^{-\alpha}$ will denote a fractional sum of order $\alpha>0$ and $\Delta_{a}^{\alpha}$ will be used to denote a fractional difference of order $\alpha>0$.

2.3. Fractional Orthonormal Legendre Polynomials. A novel class of quasi-polynomials orthogonal with respect to the fractional integration operator has been developed in [10]. The related Gaussian quadrature formulas for numerical evaluation of fractional order integrals have been proposed also in [10]. In doing so, the problem of numerical evaluation of fractional integral with unit upper integration limit (1) has been considered, by using the concept of orthogonality in the following sense:

$$
\frac{1}{\Gamma(\alpha)} \int_{0}^{1} P_{n, \beta}(\tau ; \alpha) P_{m, \beta}(\tau ; \alpha)(1-\tau)^{\alpha-1} d \tau=0,
$$

$$
n \neq m \text {, }
$$

where $P_{n, \beta}(\tau ; \alpha)=P_{n}\left(\tau^{\beta} ; \alpha\right)$ are defined in (2). We will refer to $P_{n, \beta}(\tau ; \alpha)$ as fractional Legendre polynomials. The above orthogonality relation can be written as

$$
\frac{1}{\Gamma(\alpha)} \int_{0}^{1} P_{n}\left(\tau^{\beta} ; \alpha\right) P_{m}\left(\tau^{\beta} ; \alpha\right)(1-\tau)^{\alpha-1} d \tau=0,
$$

$$
n \neq m,
$$

and by using the change of variable $x=\tau^{\beta}$, it yields

$$
\begin{aligned}
& \frac{1}{\beta \Gamma(\alpha)} \int_{0}^{1} P_{n}(x ; \alpha) P_{m}(x ; \alpha)\left(1-x^{1 / \beta}\right)^{\alpha-1} x^{(1-\beta) / \beta} d x \\
& \quad=0, \quad n \neq m .
\end{aligned}
$$

The latter relation can be read in the following sense: $\left\{P_{n}(x ; \alpha)\right\}$ is a sequence of polynomials orthogonal with respect to the positive and integrable weight function:

$$
w(x ; \alpha, \beta)=\left(1-x^{1 / \beta}\right)^{\alpha-1} x^{(1-\beta) / \beta} .
$$

From the classical theory of orthogonal polynomials [6,34], we know that there exists a unique sequence of polynomials orthogonal with respect to $w(x ; \alpha, \beta)$, up to a normalizing constant. Since

$$
\begin{array}{r}
\mu_{n}=\frac{1}{\beta \Gamma(\alpha)} \int_{0}^{1} x^{n} w(x ; \alpha, \beta) d x=\frac{\Gamma(\beta n+1)}{\Gamma(\alpha+\beta n+1)}, \\
n=0,1, \ldots,
\end{array}
$$

valid for $\alpha, \beta>0$, we have that

$$
P_{n}(x ; \alpha)=\epsilon_{n}\left|\begin{array}{ccccc}
\mu_{0} & \mu_{1} & \mu_{2} & \cdots & \mu_{n} \\
\mu_{1} & \mu_{2} & \mu_{3} & \cdots & \mu_{n+1} \\
\vdots & \vdots & \vdots & \ddots & \vdots \\
\mu_{n-1} & \mu_{n} & \mu_{n+1} & \cdots & \mu_{2 n-1} \\
1 & x & x^{2} & \cdots & x^{n}
\end{array}\right|
$$

where $\epsilon_{n}$ is the normalizing constant.

For orthonormal fractional Legendre polynomials, we have

$$
\begin{aligned}
& P_{0, \beta}(\tau ; \alpha)=\sqrt{\Gamma(\alpha+1)} \\
& P_{1, \beta}(\tau ; \alpha) \\
& =\frac{\sqrt{\Gamma(\alpha+2 \beta+1)}\left(\Gamma(\alpha+1) \Gamma(\beta+1)-\tau^{\beta} \Gamma(\alpha+\beta+1)\right)}{\sqrt{\Gamma(2 \beta+1) \Gamma^{2}(\alpha+\beta+1)-\Gamma(\alpha+1) \Gamma^{2}(\beta+1) \Gamma(\alpha+2 \beta+1)}} .
\end{aligned}
$$

Also,

$$
P_{2, \beta}(\tau ; \alpha)=\frac{\varpi_{1}}{\varpi_{2}}\left(\tau^{2 \beta} \varpi_{3}+\tau^{\beta} \varpi_{4}+\varpi_{5}\right),
$$

where

$$
\begin{aligned}
\varpi_{2} & =\Gamma(\alpha+1) \Gamma(\alpha+\beta+1) \\
\cdot & \left(\Gamma^{2}(2 \beta+1) \Gamma(\alpha+\beta+1) \Gamma(\alpha+3 \beta+1)\right. \\
- & \left.\Gamma(\beta+1) \Gamma(3 \beta+1) \Gamma^{2}(\alpha+2 \beta+1)\right), \\
\varpi_{3}= & \Gamma(\alpha+2 \beta+1) \\
& \cdot\left(\Gamma(\alpha+1) \Gamma^{2}(\beta+1) \Gamma(\alpha+2 \beta+1)\right. \\
- & \left.\Gamma(2 \beta+1) \Gamma^{2}(\alpha+\beta+1)\right) \Gamma(\alpha+3 \beta+1),
\end{aligned}
$$




$$
\begin{aligned}
\omega_{4} & =\Gamma(\alpha+\beta+1) \Gamma(\alpha+2 \beta+1) \\
\cdot & (\Gamma(3 \beta+1) \Gamma(\alpha+\beta+1) \Gamma(\alpha+2 \beta+1) \\
- & \beta \Gamma(\alpha+1) \Gamma(\beta) \Gamma(2 \beta+1) \Gamma(\alpha+3 \beta+1)), \\
\omega_{5} & =\Gamma(\alpha+1) \Gamma(\alpha+\beta+1) \\
& \cdot\left(\Gamma^{2}(2 \beta+1) \Gamma(\alpha+\beta+1) \Gamma(\alpha+3 \beta+1)\right. \\
& \left.-\Gamma(\beta+1) \Gamma(3 \beta+1) \Gamma(\alpha+2 \beta+1)^{2}\right), \\
\varpi_{1} & =\sqrt{\frac{\Gamma(\alpha+4 \beta+1)}{\omega_{6}}} \Gamma(\alpha+1) \Gamma(\alpha+\beta+1) \\
& \cdot\left(\Gamma(\beta+1) \Gamma(3 \beta+1) \Gamma^{2}(\alpha+2 \beta+1)\right. \\
& \left.-\Gamma^{2}(2 \beta+1) \Gamma(\alpha+\beta+1) \Gamma(\alpha+3 \beta+1)\right),
\end{aligned}
$$

with

$$
\begin{aligned}
\varpi_{6} & =\left(\Gamma(\alpha+1) \Gamma^{2}(\beta+1) \Gamma(\alpha+2 \beta+1)-\Gamma(2 \beta\right. \\
+ & \left.1) \Gamma(\alpha+\beta+1)^{2}\right)\left(\Gamma(4 \beta+1) \Gamma^{2}(\alpha+2 \beta+1)\right. \\
& \cdot\left(\Gamma(\alpha+1) \Gamma^{2}(\beta+1) \Gamma(\alpha+2 \beta+1)\right. \\
& \left.-\Gamma(2 \beta+1) \Gamma(\alpha+\beta+1)^{2}\right) \times \Gamma^{2}(\alpha+3 \beta+1) \\
& +\Gamma(\alpha+\beta+1)(\Gamma(\alpha+\beta+1) \\
& +\left(\Gamma(\alpha+1) \Gamma^{3}(2 \beta+1) \Gamma^{2}(\alpha+3 \beta+1)+\Gamma^{2}(3 \beta\right. \\
& \left.+1) \Gamma^{3}(\alpha+2 \beta+1)\right)-2 \Gamma(\alpha+1) \Gamma(\beta+1) \Gamma(2 \beta \\
& \left.+1) \Gamma(3 \beta+1) \Gamma^{2}(\alpha+2 \beta+1) \times \Gamma(\alpha+3 \beta+1)\right) \\
& +\Gamma(\alpha+4 \beta+1)) .
\end{aligned}
$$

From (18), it is obvious that if $\alpha, \beta \rightarrow 1$, we recover orthonormal Legendre polynomials. More precisely, for the first elements, by using their explicit expressions, we have obtained

$$
\begin{aligned}
& \lim _{\alpha, \beta \rightarrow 1} P_{0, \beta}(\tau ; \alpha)=1, \\
& \lim _{\alpha, \beta \rightarrow 1} P_{1, \beta}(\tau ; \alpha)=\sqrt{3}(1-2 \tau), \\
& \lim _{\alpha, \beta \rightarrow 1} P_{2, \beta}(\tau ; \alpha)=\sqrt{5}(6(\tau-1) \tau+1),
\end{aligned}
$$

which coincide with (6).

\section{Fractional Orthonormal Gram Polynomials}

Let $G_{n, \beta}(\tau ; \alpha, N)=G_{n}\left(\tau^{\beta} ; \alpha, N\right)$ be defined as in (2):

$$
G_{n}\left(t^{\beta} ; \alpha, N\right)=G_{n, \beta}(t ; \alpha, N)=\sum_{k=0}^{n} g_{n, k}(\alpha, N) t^{k \beta} .
$$

From (17), for $N=t-\alpha$, we define the fractional Gram orthonormal polynomials as the family which satisfies

$$
\begin{gathered}
\frac{1}{\Gamma(\alpha)} \sum_{s=0}^{N}(N+\alpha-s-1)^{(\alpha-1)} G_{n}\left(s^{\beta} ; \alpha, N\right) \\
\cdot G_{m}\left(s^{\beta} ; \alpha, N\right)=\delta_{n, m}, \quad 0 \leq n, m \leq N .
\end{gathered}
$$

Similar to the fractional Legendre case, we have that $(N+\alpha-$ $s-1)^{(\alpha-1)}$ is a positive summable weight function, of a discrete variable:

$$
\begin{aligned}
& \sum_{s=0}^{N}(N+\alpha-s-1)^{(\alpha-1)} \\
&=\frac{\Gamma(\alpha+1)}{\Gamma(N-\alpha+1) / \Gamma(N-2 \alpha+1)-\Gamma(-\alpha) / \Gamma(-2 \alpha)}, \\
& 0<\alpha<2 .
\end{aligned}
$$

It is clear from the above definition (30), and

$$
\begin{aligned}
(N+\alpha-s-1)^{(\alpha-1)}=\frac{\Gamma(\alpha+N-s)}{\Gamma(1+N-s)}, & \\
& \quad s=0,1, \ldots, N, 0<\alpha<1
\end{aligned}
$$

the following limit relation between fractional orthonormal Gram polynomials and classical orthonormal Gram polynomials defined in (8) holds:

$$
\lim _{\alpha, \beta \rightarrow 1} G_{n}\left(t^{\beta} ; \alpha, N\right)=G_{n}(t ; N), \quad n=0, \ldots, N .
$$

Moreover, by using [5, (5.11.12), p. 141] we have the following limit relation:

$$
\lim _{N \rightarrow \infty} N^{1-\alpha}(N+\alpha-N s-1)^{(\alpha-1)}=(1-s)^{\alpha-1} .
$$

Hence, we have the following limit relation between fractional orthonormal Gram polynomials and fractional orthonormal Legendre polynomials:

$$
\lim _{N \rightarrow \infty} N^{\alpha / 2} G_{n}\left(N t^{\beta}, \alpha, N\right)=P_{n, \beta}(t ; \alpha),
$$

which is indeed a fractional extension of (9). In Figure 1, we show the graphs of the first fractional orthonormal Gram polynomials.

\section{Numerical Experiments}

In this section, numerical experiments performed by using Mathematica [35] are presented.

4.1. $\alpha=3 / 5$ and $\beta=1 / 7$. In this case,

$$
\begin{aligned}
G_{1, \beta}(\tau ; \alpha, 1000) & =-0.430804 \sqrt[7]{x}+1.05357, \\
G_{1, \beta}(\tau ; \alpha, 10000) & =-0.158489 \sqrt[7]{x}+0.53835 \\
G_{1, \beta}(\tau ; \alpha, 100000) & =-0.0573041 \sqrt[7]{x}+0.270416 .
\end{aligned}
$$




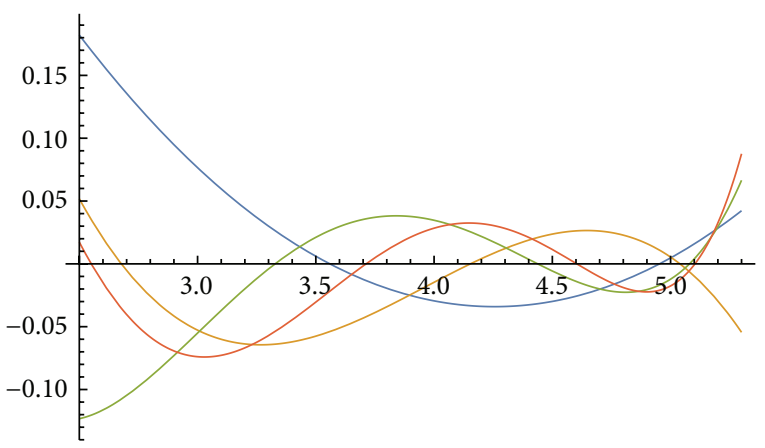

FIGURE 1: Graphs of the polynomials $G_{n, \beta}(\tau ; \alpha, N)$ after the change of variable $x=\tau^{\beta}$, for $n=2,3,4,5$, for the specific values of the parameters $\alpha=3 / 5, \beta=1 / 7$, and $N=100,000$.

Therefore,

$$
\begin{gathered}
N^{\alpha / 2} G_{1, \beta}(N \tau ; \alpha, N)=-9.18017 \sqrt[7]{x}+8.36879, \\
N=1000, \\
N^{\alpha / 2} G_{1, \beta}(N \tau ; \alpha, N)=-9.36325 \sqrt[7]{x}+8.53227, \\
N=10000, \\
N^{\alpha / 2} G_{1, \beta}(N \tau ; \alpha, N)=-9.3858 \sqrt[7]{x}+8.55131, \\
N=100000 .
\end{gathered}
$$

Notice that

$$
P_{1, \beta}(\tau ; \alpha)=-9.39005 \sqrt[7]{x}+8.55459,
$$

in accordance with (35).

Moreover,

$$
\begin{aligned}
G_{2, \beta}(\tau ; \alpha, 1000)= & 0.753333 x^{2 / 7}-3.26517 \sqrt[7]{x} \\
& +3.42283 \\
G_{2, \beta}(\tau ; \alpha, 10000)= & 0.259692 x^{2 / 7}-1.58875 \sqrt[7]{x} \\
& +2.3636 \\
G_{2, \beta}(\tau ; \alpha, 100000)= & 0.0700715 x^{2 / 7}-0.596479 \sqrt[7]{x} \\
& +1.23532 .
\end{aligned}
$$

As a consequence,

$$
\begin{aligned}
N^{\alpha / 2} G_{2, \beta}(N \tau ; \alpha, N)= & 43.0656 x^{2 / 7}-69.5788 \sqrt[7]{x} \\
& +27.1885, \quad N=1000, \\
N^{\alpha / 2} G_{2, \beta}(N \tau ; \alpha, N)= & 57.1894 x^{2 / 7}-93.8606 \sqrt[7]{x} \\
& +37.4605, \quad N=10000, \\
N^{\alpha / 2} G_{2, \beta}(N \tau ; \alpha, N)= & 59.4447 x^{2 / 7}-97.697 \sqrt[7]{x} \\
& +39.0641, \quad N=100000 .
\end{aligned}
$$

In this case,

$$
P_{2, \beta}(\tau ; \alpha)=59.7007 x^{2 / 7}-98.1278 \sqrt[7]{x}+39.2423
$$

also in accordance with (35).

4.2. $\alpha=99 / 100$ and $\beta=99 / 100$. For these values of $\alpha$ and $\beta$, we have that

$$
\begin{aligned}
G_{1, \beta}(\tau ; \alpha, 1000)= & 0.0570034 \\
& -0.000120685 x^{99 / 100}, \\
G_{1, \beta}(\tau ; \alpha, 10000)= & 0.0182768-3.9673 \\
& \cdot 10^{-6} x^{99 / 100}, \\
G_{1, \beta}(\tau ; \alpha, 100000)= & 0.00584794-1.2992 \\
& \cdot 10^{-7} x^{99 / 100} .
\end{aligned}
$$

Notice that

$$
\begin{aligned}
G_{1}(x ; 1000) & =0.0546902-0.00010938 x, \\
G_{1}(x ; 10000) & =0.0173179-3.46358 \cdot 10^{-6} x, \\
G_{1}(x ; 100000) & =0.00547714-1.0954 \cdot 10^{-7} x,
\end{aligned}
$$

in accordance with (33).

From the above expressions for fractional Gram orthonormal polynomials, we have

$$
\begin{array}{r}
N^{\alpha / 2} G_{1, \beta}(N \tau ; \alpha, N)=1.74141-3.44076 x^{99 / 100,} \\
N=1000, \\
N^{\alpha / 2} G_{1, \beta}(N \tau ; \alpha, N)=1.74542-3.45537 x^{99 / 100}, \\
N=10000, \\
N^{\alpha / 2} G_{1, \beta}(N \tau ; \alpha, N)=1.74583-3.45689 x^{99 / 100}, \\
N=100000 .
\end{array}
$$

Notice that

$$
P_{1, \beta}(\tau ; \alpha)=1.74588-3.45707 x^{99 / 100},
$$

in accordance with (35). 
Furthermore,

$$
\begin{aligned}
G_{2, \beta}(\tau ; \alpha, 1000)= & 4.9936 \cdot 10^{-7} x^{99 / 50} \\
& -0.000469348 x^{99 / 100} \\
& +0.073817 \\
G_{2, \beta}(\tau ; \alpha, 10000)= & 1.68426 \cdot 10^{-9} x^{99 / 50} \\
& -0.0000154412 x^{99 / 100} \\
& +0.0237096, \\
G_{2, \beta}(\tau ; \alpha, 100000)= & .64578 \cdot 10^{-12} x^{99 / 50}-5.05717 \\
& \cdot 10^{-7} x^{99 / 100}+0.00758763, \\
G_{2}(x ; 1000)= & 4.2320 \cdot 10^{-7} x^{2} \\
& -0.000423206 x \\
& +0.0704639, \\
& 1.34130 \cdot 10^{-9} x^{2} \\
& -0.0000134131 x \\
& +0.0223529, \\
G_{2}(x ; 10000)= & 4.24253 \cdot 10^{-12} x^{2}-4.24253 \\
& \cdot 10^{-7} x+0.00707082, \\
G_{2}(x ; 100000)= &
\end{aligned}
$$

in accordance with (33).

By using the above expressions for fractional Gram orthonormal polynomials, we obtain

$$
\begin{aligned}
& N^{\alpha / 2} G_{2, \beta}(N \tau ; \alpha, N)= 13.2866 x^{99 / 50} \\
&-13.3812 x^{99 / 100} \\
&+2.25505, \quad N=1000 \\
& N^{\alpha / 2} G_{2, \beta}(N \tau ; \alpha, N)= 13.3786 x^{99 / 50} \\
&-13.4487 x^{99 / 100} \\
&+2.26425, \quad N=10000, \\
& N^{\alpha / 2} G_{2, \beta}(N \tau ; \alpha, N)= 13.3882 x^{99 / 50} \\
&-13.4557 x^{99 / 100}+2.2652, \\
& N=100000 .
\end{aligned}
$$

Notice that

$$
\begin{aligned}
P_{2, \beta}(\tau ; \alpha)= & 13.3894 x^{99 / 50}-13.4566 x^{99 / 100} \\
& +2.2653
\end{aligned}
$$

in accordance with (35).
4.3. Interlacing Properties of the Zeros. Let us consider the polynomials obtained from $G_{n}(x ; \alpha, \beta, N)=G_{n, \beta}(\tau ; \alpha, N)$ after the change of variable $x=\tau^{\beta}$. As it happens in the classical discrete situation [8], we have observed numerically that their zeros are real and simple, they lie in the interval $[0, N]$, and there exists at most one zero in each interval $[i, i+1], i=0, \ldots, N-1$.

As an example, for the specific values of the parameters $\alpha=3 / 5, \beta=1 / 7$, and $N=100,000$, we have obtained the following zeros of the above mentioned polynomials:

$$
\begin{aligned}
& G_{2}(x ; \alpha, \beta, N)=0, \quad\{3.55906,4.95338\}, \\
& G_{3}(x ; \alpha, \beta, N)=0, \quad\{2.67741,4.15339,5.03948\}, \\
& G_{4}(x ; \alpha, \beta, N)=0,
\end{aligned}
$$

$\{1.93655,3.32757,4.43799,5.07999\}$,

$$
\begin{aligned}
& G_{5}(x ; \alpha, \beta, N)=0, \\
& \quad\{0.978606,2.55054,3.71232,4.60351,5.10308\} .
\end{aligned}
$$

\section{Conclusions}

By using fractional differences, we have introduced fractional Gram orthonormal polynomials. These polynomials converge as $N \rightarrow \infty$ to fractional Legendre orthonormal polynomials. Moreover, as $\alpha, \beta \rightarrow 1$, they converge to "classical" Gram orthonormal polynomials. Finally, as $N \rightarrow$ $\infty$ and $\alpha, \beta \rightarrow 1$, fractional Gram orthonormal polynomials converge to shifted Legendre orthonormal polynomials. We conjecture, in view of our numerical computations, that the zeros are real and simple and there exists at most one zero inside each of the intervals $[i, i+1], i=0,1, \ldots, N-1$.

\section{Conflict of Interests}

The authors declare no conflict of interests.

\section{Authors' Contribution}

Each of the authors, I. Area, J. Losada, Juan J. Nieto, and J. D. Djida, contributed to each part of this study equally and read and approved the final version of the paper.

\section{Acknowledgments}

The referee deserves special thanks for careful reading of the paper. The work of I. Area has been partially supported by the Ministerio de Economía y Competitividad of Spain under Grant MTM2012-38794-C02-01 and cofinanced by the European Community Fund FEDER. Juan J. Nieto and J. Losada also acknowledge partial financial support by the Ministerio de Economía y Competitividad of Spain under Grants MTM2010-15314 and MTM2013-43014-P and XUNTA under Grant R2014/002 and cofinanced by the European Community Fund FEDER. The first author also thanks the hospitality of the African Institute for Mathematical Sciences Cameroon, AIMS, where the essential part of this research was performed during his visits in November 2014, May 2015, and June 2015. 


\section{References}

[1] B. Ross, "Fractional calculus," Mathematics Magazine, vol. 50, no. 3, pp. 115-122, 1977.

[2] G. W. Leibniz, "Letter from Hanover, Germany; to G.F.A. L'Hôpital," in Mathematische Schriften 1849, vol. 2, pp. 301-302, Olms, Hidesheim, Germany, 1695, reprinted in 1962.

[3] C. Li, F. Zeng, and F. Liu, "Spectral approximations to the fractional integral and derivative," Fractional Calculus and Applied Analysis, vol. 15, no. 3, pp. 383-406, 2012.

[4] A. M. Legendre, "Sur l'attraction des sphéroïdes homogénes," Mémoires de Mathématique et de Physique, Présentés à l'Académie Royale des Sciences, par Divers Savans, et lus dans ses Assemblées, vol. 10, pp. 411-434, 1785.

[5] NIST Digital Library of Mathematical Functions, Release 1.0.8, 2014, http://dlmf.nist.gov/.

[6] M. E. H. Ismail, Classical and Quantum Orthogonal Polynomials in One Variable, Cambridge University Press, Cambridge, UK, 2005.

[7] R. Koekoek, P. A. Lesky, and R. F. Swarttouw, Hypergeometric Orthogonal Polynomials and Their q-Analogues, Springer, Berlin, Germany, 2010.

[8] A. F. Nikiforov, S. K. Suslov, and V. B. Uvarov, Classical Orthogonal Polynomials of a Discrete Variable, Springer, Berlin, Germany, 1991.

[9] F. W. J. Olver, D. W. Lozier, R. F. Boisvert, and C. W. Clark, Eds., NIST Handbook of Mathematical Functions, Cambridge University Press, New York, NY, USA, 2010.

[10] M. R. Rapaić, T. B. Šekara, and V. Govedarica, "A novel class of fractionally orthogonal quasi-polynomials and new fractional quadrature formulas," Applied Mathematics and Computation, vol. 245, pp. 206-219, 2014.

[11] I. Area, J. Losada, and A. Manintchap, "On some fractional Pearson equations," Fractional Caculus and Applied Analysis, vol. 18, no. 5, 2015.

[12] P. L. Chebyshev, "Sur l'interpolation des valeurs équidistantes," Mémoires de l'Académie Impériale des Sciences de Saint Pétersbourg, vol. 25, no. 5, pp. 219-242, 1875, Oeuvres, vol. 2, 219-242.

[13] A. B. J. Kuijlaars and E. A. Rakhmanov, "Zero distributions for discrete orthogonal polynomials," Journal of Computational and Applied Mathematics, vol. 99, no. 1-2, pp. 255-274, 1998.

[14] I. Area, D. K. Dimitrov, E. Godoy, and V. Paschoa, "Approximate calculation of sums I: bounds for the zeros of Gram polynomials," SIAM Journal on Numerical Analysis, vol. 52, no. 4, pp. 1867-1886, 2014.

[15] A. Erdélyi, W. Magnus, F. Oberhettinger, and F. G. Tricomi, Higher Transcendental Functions, vol. 2, Robert E Krieger Publishing, Melbourne, Fla, USA, 1981.

[16] G. Szegö, Orthogonal Polynomials, American Mathematical Society, Providence, RI, USA, 4th edition, 1975.

[17] J. P. Gram, "Ueber die Entwickelung reeller Functionen in Reihen mittelst der Methode der kleinsten Quadrate," Journal für die reine und angewandte Mathematik, vol. 1883, no. 94, pp. 41-73, 2009.

[18] J. Baik, T. Kriecherbauer, K. T.-R. McLaughlin, and P. D. Miller, Discrete Orthogonal Polynomials, vol. 164 of Annals of Mathematics Studies, Princeton University Press, Princeton, NJ, USA, 2007.

[19] G. Dahlquist and Å. Björck, Numerical Methods in Scientific Computing, vol. 1, Society for Industrial and Applied Mathematics (SIAM), Philadelphia, Pa, USA, 2008.
[20] A. A. Kilbas, H. M. Srivastava, and J. J. Trujillo, Theory and Applications of Fractional Differential Equations, vol. 204, Elsevier Science, Amsterdam, The Netherlands, 2006.

[21] K. S. Miller and B. Ross, An Introduction to the Fractional Calculus and Fractional Differential Equations, John Wiley \& Sons, New York, NY, USA, 1993.

[22] K. B. Oldham and J. Spanier, The Fractional Calculus, Academic Press, New York, NY, USA, 1974.

[23] S. Samko, A. A. Kilbas, and O. Marichev, Fractional Integrals and Derivatives, Taylor \& Francis, 1993.

[24] F. M. Atici, A. Cabada, and J. B. Ferreiro, "First order difference equations with maxima and nonlinear functional boundary value conditions," Journal of Difference Equations and Applications, vol. 12, no. 6, pp. 565-576, 2006.

[25] F. M. Atici and P. W. Eloe, "A transform method in discrete fractional calculus," International Journal of Difference Equations, vol. 2, no. 2, pp. 165-176, 2007.

[26] J. Cermak and T. Kisela, "Note on a discretization of a linear fractional differential equation," Mathematica Bohemica, vol. 135, no. 2, pp. 179-188, 2010.

[27] J. Čermák, T. Kisela, and L. Nechvátal, "Discrete Mittag-Leffler functions in linear fractional difference equations," Abstract and Applied Analysis, vol. 2011, Article ID 565067, 21 pages, 2011.

[28] K. S. Miller and B. Ross, "Fractional difference calculus," in Univalent Functions, Fractional Calculus, and Their Applications (Kōriyama, 1988), Ellis Horwood Series in Mathematics and Its Applications, pp. 139-152, Horwood, Chichester, UK, 1989.

[29] M. D. Ortigueira, "Fractional central differences and derivatives," Journal of Vibration and Control, vol. 14, no. 9-10, pp. 1255-1266, 2008.

[30] J. Tariboon, S. K. Ntouyas, and P. Agarwal, "New concepts of fractional quantum calculus and applications to impulsive fractional q-difference equations," Advances in Difference Equations, vol. 2015, article 18, 2015.

[31] I. Podlubny, Fractional Differential Equations, vol. 198 of Mathematics in Science and Engineering, Academic Press, 1999.

[32] T. Abdeljawad, D. Baleanu, F. Jarad, and R. P. Agarwal, "Fractional sums and differences with binomial coefficients," Discrete Dynamics in Nature and Society, vol. 2013, Article ID 104173, 6 pages, 2013.

[33] J. Diblík, M. Fećkan, and M. Posipíšil, "Nonexistence of periodic solutions and $S$-asymptotically periodic solutions in fractional difference equations," Applied Mathematics and Computation, vol. 257, pp. 230-240, 2015.

[34] T. S. Chihara, An Introduction to Orthogonal Polynomials, Gordon and Breach Science Publishers, New York, NY, USA, 1978.

[35] Wolfram Research Inc, Mathematica, version 10.0.2.0, Wolfram Research Inc, Champaign, Ill, USA, 2010. 


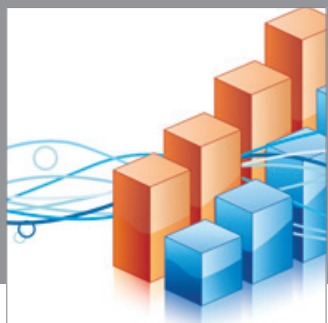

Advances in

Operations Research

mansans

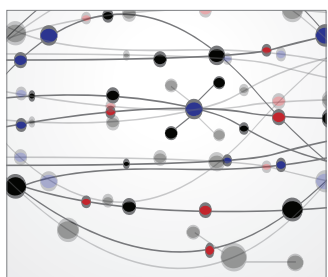

The Scientific World Journal
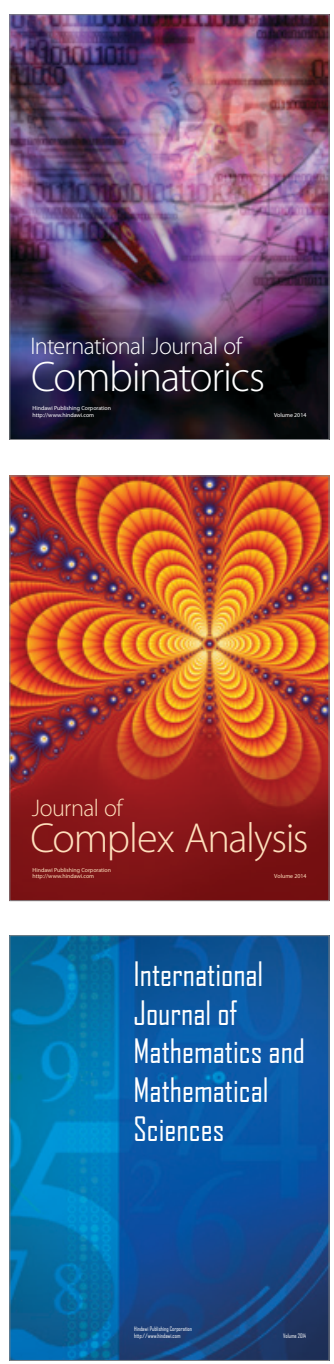
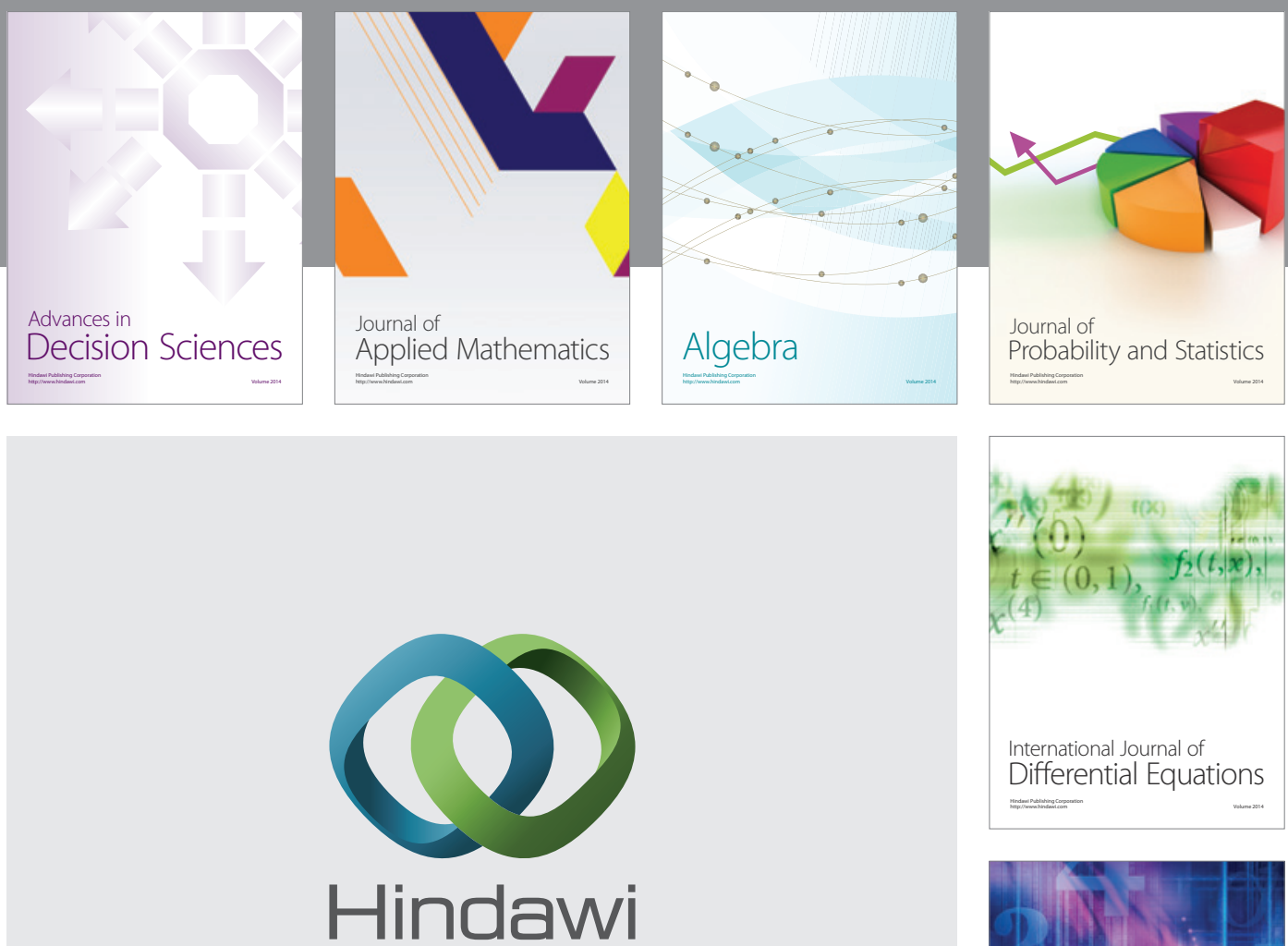

Submit your manuscripts at http://www.hindawi.com
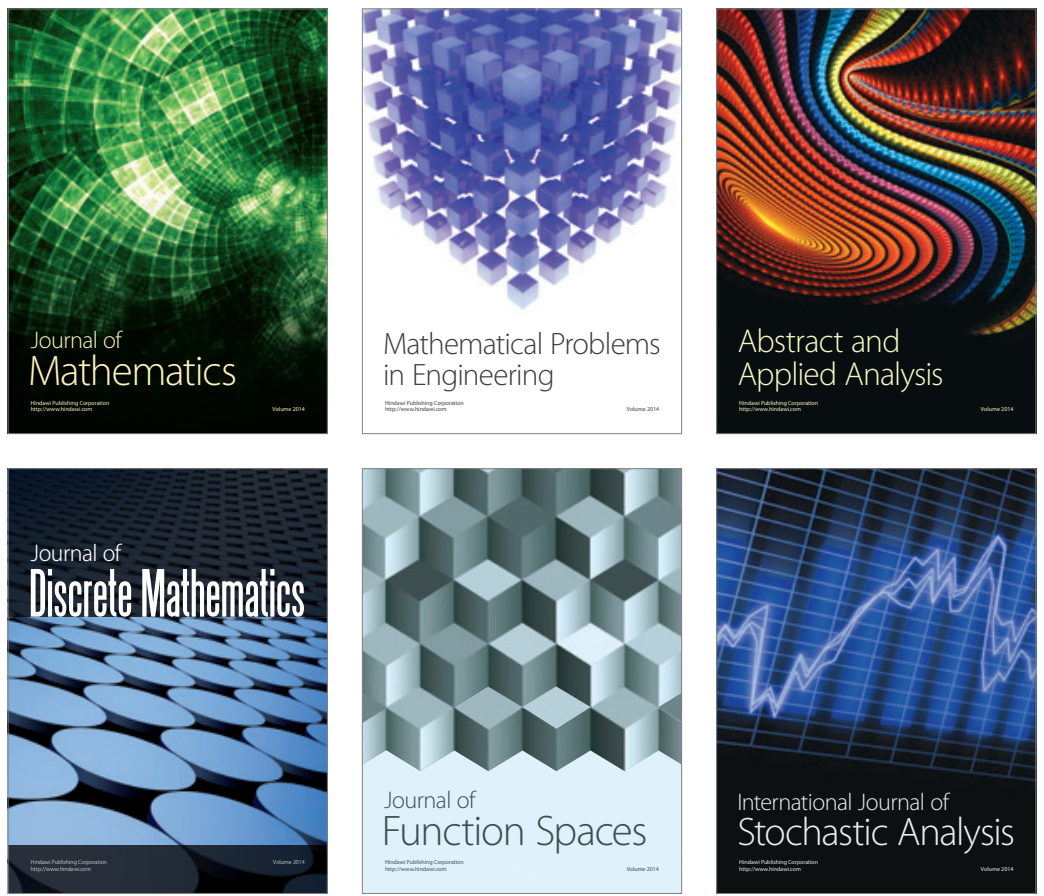

Journal of

Function Spaces

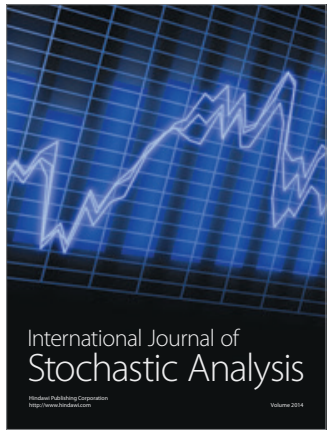

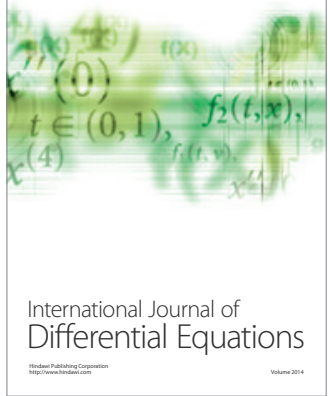
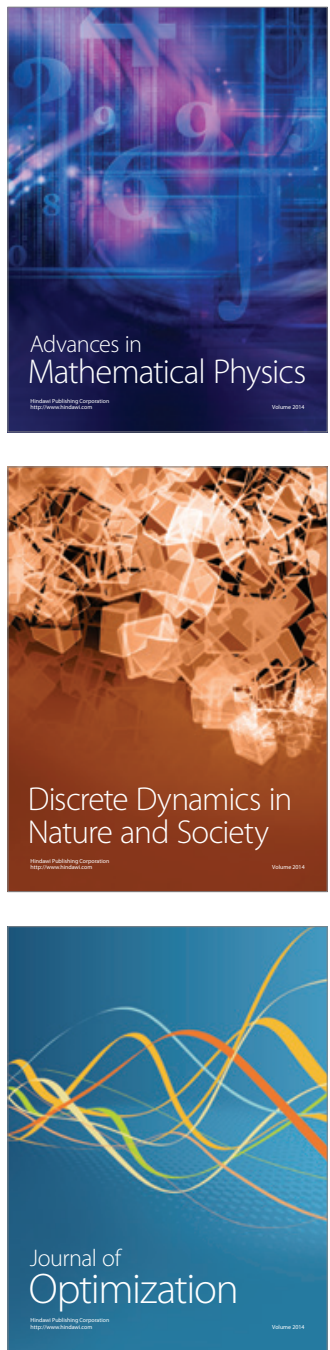